\title{
Sarcoidosis: muchas caras, una enfermedad. Revisión narrativa de la literatura
}

\author{
Andrés Chavarriaga-Restrepo ${ }^{1}$, Juan Esteban López-Amaya² ${ }^{2}$ Miguel Antonio Mesa- Navas³ \\ Carlos Jaime Velásquez-Franco ${ }^{4}$
}

\section{RESUMEN}

La sarcoidosis es una enfermedad granulomatosa sistémica de etiología desconocida. Esta puede afectar a pacientes de todas las latitudes y edades, siendo más frecuente entre la tercera y cuarta década de la vida con un segundo pico alrededor de los 50 años en las poblaciones escandinava y japonesa. Es más frecuente en mujeres y grave en la población afrodescendiente. Los antígenos que inician esta respuesta granulomatosa son desconocidos, pero se presume que son aerotransportados por la alta frecuencia de compromiso pulmonar en esta enfermedad. Su presentación clínica abarca una amplia gama de manifestaciones, desde formas agudas y limitadas hasta el compromiso crónico con daño orgánico progresivo y muerte. Su diagnóstico se basa en la existencia de los granulomas no caseificantes en los tejidos, con la exclusión de otras enfermedades, entre ellas infección por micobacterias.

\section{PALABRAS CLAVE}

Linfadenopatía; PuImón; Sarcoidosis

\section{SUMMARY}

Sarcoidosis: many faces, one disease. A narrative review

Internista, Clínica CES, Medellín, Colombia.

Radiólogo, Clínica Universitaria Bolivariana, Medellín, Colombia.

Docente, Escuela de Ciencias de la Salud Universidad Pontificia Bolivariana, Medellín, Colombia.

Profesor de Medicina Interna, Escuela de Ciencias de la Salud. Universidad Pontificia Bolivariana, Sección de Reumatología. Clínica Universitaria Bolivariana. Medellín, Colombia.

Correspondencia: Andrés Chavarriaga-Restrepo; andresch_1@hotmail.com

Recibido: marzo 31 de 2018

Aceptado: agosto 31 de 2018

Cómo citar: Chavarriaga-Restrepo A, López-Amaya JE, Mesa-Navas MA, Velásquez-Franco CJ. Sarcoidosis: muchas caras, una enfermedad. Revision narrativa de la literatura. latreia. 2019 Jul-Sep;32(3):191-203. D0I 10.17533/udea.iatreia.11. 
Sarcoidosis is a systemic granulomatous disease of unknown etiology. It affects patients from all the latitudes and ages, being more frequent between the third and the fourth decades of life and with a second peak around the sixth decade of age in the Japanese and Scandinavian population. It's more common in women, and it has a more severe course in Afro-descendant population. The antigens that start the granulomatous response are unknown, but presumed that be airborne antigens, because of the high frequency of pulmonary involvement. Its clinical presentation is variable, ranging from acute self-limited presentation to chronic disease with progressive organ damage and death. Its diagnosis relies upon the demonstration of noncaseating granulomas in the affected tissue and the exclusion of other conditions including mycobacteria infections.

\section{KEY WORDS}

Lung; Lymphadenopathy; Sarcoidosis

\section{INTRODUCCIÓN}

La sarcoidosis es una enfermedad sistémica granulomatosa de etiología desconocida y distribución global (1). Su diagnóstico se basa en la exclusión de otras enfermedades que puedan causar granulomas con necrosis sin caseificación, sumado al compromiso de al menos dos órganos, en un paciente con síntomas compatibles (2).

Su origen se remonta a 1899, cuando Boeck dio el nombre a múltiples lesiones sarcoidales benignas en las regiones extensoras de los miembros superiores de un policía de 36 años. En el estudio microscópico se encontró la presencia de células epitelioides con un núcleo grande y pálido, asociado a algunas células gigantes que tenían apariencia de sarcoma, razón por la cual se dio dicho nombre en ese entonces y es conservado hasta nuestros días (3).

En los últimos años se ha producido un avance en el conocimiento sobre la fisiopatología, las manifestaciones clínicas y el tratamiento de la enfermedad, durante la siguiente revisión abordaremos algunos de ellos.

\section{EPIDEMIOLOGÍA}

Desde el punto de vista epidemiológico, la sarcoidosis puede afectar a pacientes de todas las latitudes y edades, es más frecuente entre la tercera y cuarta década de la vida, con un segundo pico alrededor de los 50 años en las poblaciones escandinava y japonesa (4, 5). Es 1,5 veces más frecuente en mujeres (5), con una prevalencia reportada entre 1-60 de 100.000 pacientes que varía según la localización y la raza de las distintas series reportadas, pues es mayor en blancos y afrodescendientes, siendo tres veces más frecuente en la segunda de ellas como se muestra en la Tabla 1 (6).

Tabla 1. Prevalencia por raza

\begin{tabular}{cc}
\hline Población/Raza & Prevalencia \\
\hline Estados Unidos & $10-40 / 100.000$ \\
\hline Afroamericanos & $35,4-60 / 100.000$ \\
\hline Blancos & $10,4-14 / 100.000$ \\
\hline Escandinavos & $50-60 / 100.000$ \\
\hline Japón & $1-2 / 100.000$ \\
\hline Argentina/Brasil & $1,5 / 100.000$ \\
\hline
\end{tabular}

Fuente: referencia 18

Es importante resaltar, además, que existe variación no solo en la prevalencia de la enfermedad si no en su forma de presentación de acuerdo con la raza, siendo más frecuentes las formas crónicas y el compromiso ocular en la población afrodescendiente y las formas asintomáticas en la población blanca; impactando de este modo el pronóstico de la enfermedad, pues la población afroamericana presenta mayor requerimiento de esteroides y menos éxito en el desmonte de estos. Otra variación descrita se observa en Japón, donde existe una mayor incidencia de un compromiso cardiaco descrito en estudios de autopsia, presente hasta en el $50 \%$ de los pacientes y es la principal causa de muerte $(4,5,7)$.

En la epidemiologia local, se encuentra una serie de casos del nororiente colombiano (8) donde se incluyeron 8 pacientes, $50 \%$ hombres con promedio de edad de 40 años, siendo el síntomas más frecuente de la serie la disnea $75 \%$, seguido por tos y pérdida de peso. El compromiso pulmonar se observó en un $50 \%$ 
de los pacientes, siendo característicamente el compromiso bilateral y de lóbulos superiores (8). También se encuentra la serie de Muñoz et al en Medellín con 22 pacientes, de los cuales $89 \%$ eran mujeres, con una mediana de edad de 46 años, siendo la principal causa de consulta los síntomas cutáneos y articulares, con una mediana de tiempo de los síntomas de 5 meses al diagnóstico. Catorce pacientes tenían síntomas pulmonares, 14 tenía síntomas en la piel determinados por eritema nodoso, pápulas, cambios en tatuaje y síndrome de Sweet; en el 62 \% se reportaron síntomas articulares (9).

\section{FISIOPATOLOGÍA}

Si bien la fisiopatología de la sarcoidosis no es completamente clara, se ha considerado que su etiología puede ser disparada por un agente medio ambiental, un antígeno que en un huésped susceptible desencadena esta reacción granulomatosa. Con respecto al antígeno inicial su identidad es desconocida, pero se presume es aerotransportado, pues se presenta algún tipo de compromiso pulmonar en el $95 \%$ de los casos (10). Tratando de identificar este antígeno, se han realizado diversos estudios en los cuales se ha encontrado de forma variable material genético en los granulomas, principalmente de micobacterias (11). Lo anterior sustentado de un estudio realizado en Johns Hopkins en el cual se comparó 9 pacientes con sarcoidosis y 14 controles sin la enfermedad, encontrando anticuerpos monoclonales anticatalasa peroxidasa de M. tuberculosis en el $55 \%$ de las muestras evaluadas en el grupo de sarcoidosis y en ninguna del grupo de control (12).

En el estudio de casos y controles para conocer la etiología de la sarcoidosis (ACCESS) (13), que reclutó a 736 pacientes, se encontraron otros factores desencadenantes asociados con ciertas ocupaciones (crianza de aves, fabricación de automóviles, jardinería) y exposiciones ambientales (insecticidas, aves, algodón, radiación, polvo orgánico, material de construcción), lo que refuerza el concepto de que en un paciente con susceptibilidad genética, una variedad de antígenos puede desencadenar la enfermedad. Finalmente, el bajo estatus socioeconómico también se ha asociado al desarrollo de esta enfermedad, en parte explicado por el pobre acceso a los servicios de salud $y$ en parte por la tendencia en este grupo de personas a desempeñar trabajos con mayor exposición a factores desencadenantes $(14,15)$.

Como enfermedad tiene un componente inflamatorio sistémico, el cual se presume es debido a la presentación antigénica a través del complejo mayor de histocompatibilidad con activación de los linfocitos Th1. Estos infiltran el pulmón y producen citoquinas como el factor de necrosis tumoral alfa (TNF- $\alpha$ ), el factor de crecimiento transformante beta (TGF- $\beta$ ) y las interleucinas 2 y 12 , entre otras; lo que lleva a la formación de granulomas con necrosis sin caseificación y con daño de la microarquitectura local (16).

Los granulomas están compuestos por linfocitos T CD4 (Th1) con macrófagos activados, los cuales simulan una célula epitelioide (por lo que se llaman granulomas epitelioides), rodeados a su vez por fibroblastos, células B y linfocitos T CD8 (4). Estos granulomas epitelioides son la fuente principal de enzima convertidora de angiotensina, descrita incluso como ayuda diagnóstica en la enfermedad.

La formación de granulomas es uno de los ejes centrales de la enfermedad y consta de 4 estadios: la iniciación donde se reclutan macrófagos y monocitos, los primeros interiorizan el antígeno, el cual procesan $y$ presentan, mediante el complejo mayor de histocompatibilidad tipo II a los linfocitos T CD4. Posteriormente, se da la fase de acumulación donde existe una proliferación oligoclonal con diferenciación de los linfocitos CD4 y un aumento en el número de linfocitos CD-4, CD-8 y monocitos; seguida de una fase efectora con participación de varios mediadores como las interleucinas 2 y 12 y el interferón gamma (IFN- $\gamma$ ). Finalmente, el destino del granuloma se define con base en el perfil de citoquinas que estén presentes en ese momento, de tal forma que si predomina el aclaramiento antigénico y la IL-10, se cambiara la vía hacia una respuesta tipo Th2 con una subsecuente limitación de la enfermedad, y en caso de predominar el factor de crecimiento transformante beta y la catelicidina 18 , se perpetuará el proceso fibrótico que a la larga dará cuenta del daño en la arquitectura y la disfunción de los órganos afectados (Figura 1) $(17,18)$. 


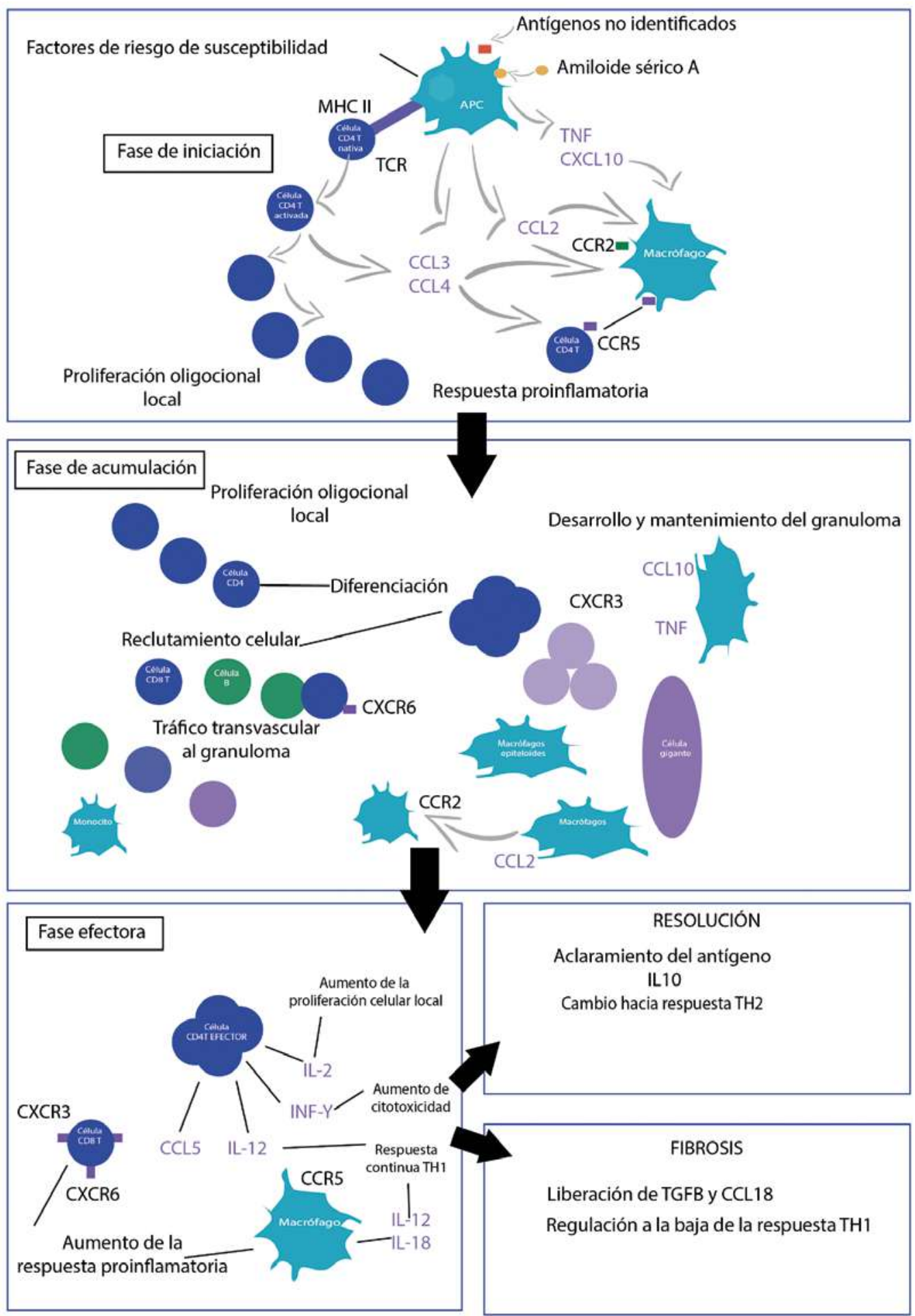

Figura 1. Fases de la fisiopatogénesis. Fuente: referencia 6. 


\section{PRESENTACIÓN CLÍNICA}

Como enfermedad sistémica, puede afectar cualquier órgano con un espectro variable de presentación desde las formas asintomáticas, pasando por las formas autolimitadas de curso inherentemente benigno hasta las formas crónicas con daño orgánico acumulado y un peor pronóstico (16). Con respecto a las formas asintomáticas, son usualmente diagnosticadas de forma incidental durante la realización de una imagen torácica, donde se encuentran los hallazgos característicos de la enfermedad, dados por adenopatías hiliares simétricas; siendo adenopatías unilaterales o ausentes, en el $5 \%$ de los casos (6).

Las formas agudas son representadas de forma prototípica por el síndrome de Löforen, dado por la triada linfadenopatía hiliar bilateral, eritema nodoso y artritis bilateral de tobillo. Esta, junto con la fiebre uveoparotídea (síndrome de Heerfordt), constituyen los dos escenarios sintomáticos en los que es posible realizar el diagnóstico de sarcoidosis sin confirmación histológica (19).

El síndrome de Löfogren tiene una distribución similar entre hombres y mujeres, un pico de incidencia entre los 30 y 40 años y un segundo pico de presentación en mujeres entre los 45 y 65 años. Usualmente no requiere tratamiento pues se autoresuelve, lo cual habla de su buen pronóstico, siendo una forma de manifestación inusual en la población afroamericana (16).

El síndrome de Heerfordt es también una manifestación de buen pronóstico y consiste en la presencia de uveítis y parotiditis bilateral, presentes en el $73 \%$ de los casos. Los otros síntomas característicos que completan este síndrome y son menos frecuentes son la fiebre y la parálisis facial (20).

Las formas crónicas tienen un peor pronóstico y son más frecuentes en la raza negra, el órgano principalmente afectado es el pulmón (90-95\%), este puede presentar un amplio abanico de síntomas que van desde tos, disnea, sibilancias ocasionales, hemoptisis y dolor torácico, hasta el compromiso del parénquima pulmonar con fibrosis $(2,21)$. Esta afección es la que dictamina el pronóstico de la enfermedad en la población no japonesa, por la pobre supervivencia que se asocia a las formas avanzadas (22). Otros sistemas comprometidos en orden de frecuencia se muestran en la Tabla 2. Con respecto al compromiso multisistémico de la enfermedad, el estudio ACCESS (13) encontró que el $50 \%$ de los pacientes tenían el compromiso de un órgano, $30 \%$ dos órganos y $20 \%$ en tres o más órganos.

Tabla 2. Compromiso por órganos

\begin{tabular}{|cccc|}
\hline Cohorte & ACCESS (13) & MUSC (23) & TTS (24) \\
\hline Número & 736 & 1.582 & 293 \\
\hline Pulmón \% & 95 & 89 & 99 \\
\hline Piel \% & 16 & 32 & 16 \\
Ojo \% & 12 & 23 & 5 \\
\hline $\begin{array}{c}\text { Linfadenopatías } \\
\text { periféricas \% }\end{array}$ & 15 & 12 & 13 \\
\hline Hígado \% & 12 & 20 & - \\
\hline Bazo \% & 7 & 7 & - \\
\hline Neurológico \% & 5 & 9 & 3 \\
\hline
\end{tabular}

Fuente: adaptada de referencia 22

\section{HALLAZGOS RADIOLÓGICOS}

Hace más de 40 años Siltzbach desarrolló un sistema de clasificación para la sarcoidosis basado en un patrón de hallazgos radiológicos que aún es ampliamente usado y tiene valor pronóstico. Esta clasificación define 5 estadios de sarcoidosis resumidos en la Tabla 3. Cada estadio se relaciona con una probabilidad de remisión espontánea, siendo de un 60-90 \% en los pacientes con enfermedad estadio 1, 40-70 \% con enfermedad estadio 2 (Figura 2), 10-20\% de pacientes con enfermedad estadio 3 y $0 \%$ de pacientes con estadio $4(23,24)$.

\section{Tabla 3. Estadios de sarcoidosis basados en los hallazgos radiológicos}

\begin{tabular}{lcc}
\hline Estadio & Hallazgo & $\begin{array}{c}\text { Frecuencia de } \\
\text { presentación \% }\end{array}$ \\
\hline Estadio 0 & Normal & $5-10$ \\
Estadio 1 & Linfadenopatías & 50 \\
\hline Estadio 2 & $\begin{array}{c}\text { Linfadenopatías e infiltrados } \\
\text { pulmonares }\end{array}$ & $25-30$ \\
\hline Estadio 3 & Infiltrados pulmonares & $10-12$ \\
\hline Estadio 4 & Fibrosis & 5 \\
\hline
\end{tabular}


Clasificación de sarcoidosis basada en los hallazgos radiográficos. Los porcentajes indican la proporción de pacientes con diagnóstico de sarcoidosis y su respectivo estadio en el momento de la presentación de la enfermedad.
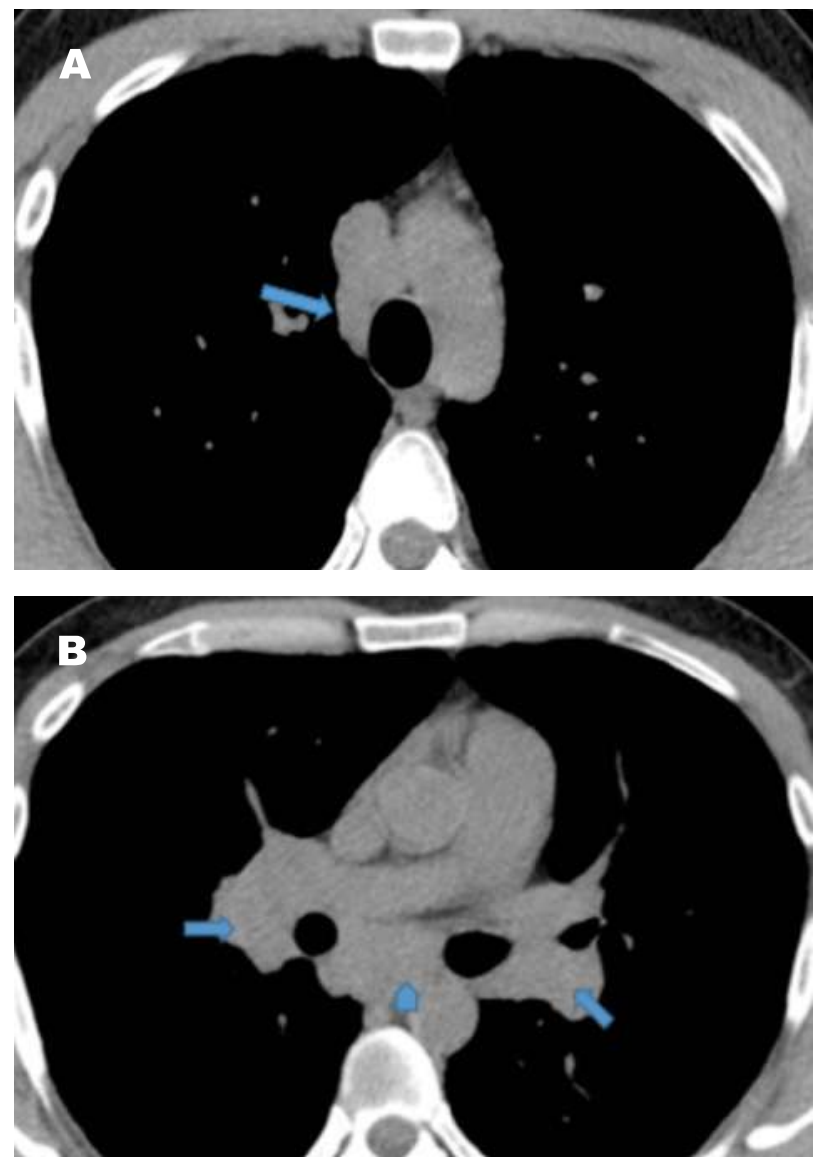

Figura 2. 2A. Tomografía computarizada de alta resolución en ventana de tejido blando mostrando adenopatía paratraqueal derecha. 2B. Muestra adenopatías hiliares, bilaterales y simétricas (flechas rectas) y conglomerado de adenopatias subcarinales (cabeza de flecha). Fuente: imagen del grupo de reumatología de la Clínica Universitaria Bolivariana.

Este sistema de clasificación fue desarrollado antes de la introducción de la tomografía computarizada (TC) y la información recolectada hasta el día de hoy con respecto al pronóstico está basada en los hallazogos en radiografía, no en TC. La TC de alta resolución es más sensible que la radiografía en la representación de anormalidades sutiles del parénquima pulmonar en un estadio temprano de la enfermedad, aún en el estadio $1(21,25)$; sin embargo, el valor del pronóstico para los hallazoos tomográficos aún no se ha estudiado ampliamente (26).

\section{HALLAZGOS EN LA TOMOGRAFÍA COMPUTARIZADA DE ALTA RESOLUCIÓN}

La TC de alta resolución es superior a la convencional en la evaluación y diferenciación entre inflamación y fibrosis en pacientes con sarcoidosis pulmonar $(27,28)$. Su mejor resolución espacial permite mejorar la detección de opacidades reticulares, nodulares, engrosamiento de septos interlobulares y opacidades en vidrio deslustrado. La TC de alta resolución también tiene mayor utilidad en la diferenciación entre inflamación activa y fibrosis irreversible en pacientes que presentan la enfermedad en el estadio 2 o 3. Los nódulos, las opacidades en vidrio deslustrado y las opacidades alveolares sugieren una inflamación granulomatosa que puede ser reversible con terapia (29). Por el contrario, los quistes en panal de abejas, las bandas septales gruesas, distorsión de la arquitectura, pérdida de volumen $y$ bronquiectasias de tracción indican una fibrosis irreversible. La tomografía de alta resolución también puede ser útil para comprobar el diagnóstico en pacientes con manifestaciones clínicas atípicas o que presenten hallazgos radiológicos inusuales (30).

La presentación más común de la sarcoidosis corresponde a la presencia de adenopatías paratraqueales derechas e hiliares bilaterales y simétricas. Las adenopatías hiliares bilaterales, solas o en combinación con adenopatías mediastinales, se presentan aproximadamente en un $95 \%$ de los pacientes con sarcoidosis $(25,31)$; las adenopatías paratraqueales izquierdas, subcarinales, ventana aortopulmonar y prevasculares están presentes hasta en $50 \%$ de los pacientes (28). Las calcificaciones de las adenopatías están directamente relacionadas con la duración de la enfermedad granulomatosa. Se ven en el $3 \%$ de los pacientes después de 5 años y en el $20 \%$ después de 10 años; pueden tener varias apariencias: amorfas, punteadas, forma de crispeta o en cáscara de huevo.

En cuanto a la afectación del parénquima pulmonar, lo característico es la distribución perilinfática de lesiones micronodulares (del 75-90 \% de los casos), con una distribución bilateral y simétrica predominantemente, 
aunque no de forma invariable en las zonas medias y superiores. Estos nódulos se encuentran usualmente en el intersticio peribroncovascular, subpleurales $y$ septos interlobulillares, pueden coalescer en el tiempo formando lesiones de mayor tamaño (macronódulos) (Figura 3). Los granulomas sarcoides también causan con frecuencia engrosamiento nodular o irregular del intersticio peribroncovascular (29, 30).

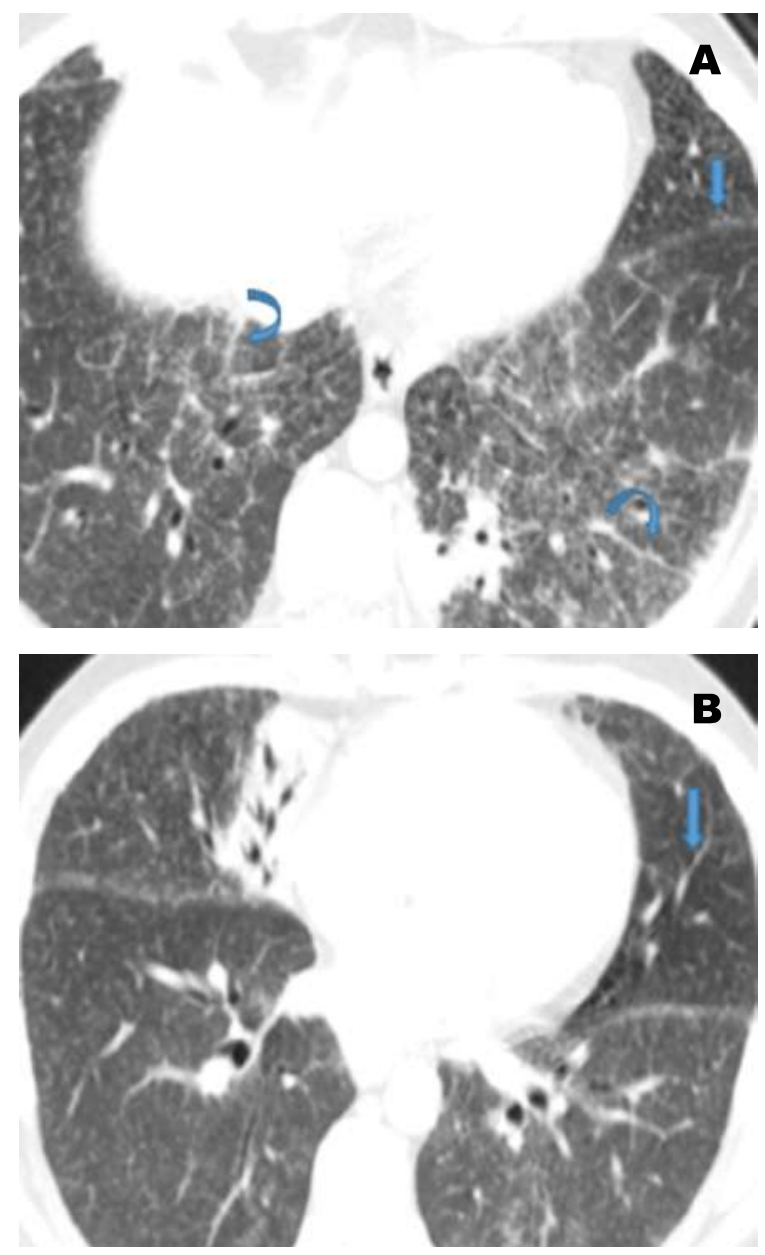

Figura 3. 3A. TC de alta resolucion en inspiracion donde se evidencia micronodulos de distribución perilinfática. Se demuestra el compromiso en las cisuras (flecha recta) y el engrosamiento nodular de septos interlobulillares (flechas curvas). 3B. Opacidades en vidrio deslustrado (atenuacion normal del parenquima pulmonar senalada por la flecha recta en la lingula). Tambien se muestran micronodulos de distribucion perilinfatica y engrosamiento nodular de septos interlobulillares que tienen mejor representacion en la $3 \mathrm{~A}$. Fuente: imagen del grupo de reumatología de la Clínica Universitaria Bolivariana.
Las opacidades en vidrio deslustrado se presentan en aproximadamente el $40 \%$ de los pacientes y se originan por la confluencia de múltiples lesiones intersticiales granulomatosas micronodulares y fibróticas, Io que genera compresión pero no el llenado del espacio aéreo visto en la alveolitis $(25,27)$. El patrón de atenuación en mosaico en pacientes con sarcoidosis es resultado del compromiso de la pequeña vía aérea por granulomas o fibrosis, lo cual puede originar una obstrucción y asociarse al atrapamiento aéreo, este último es mejor visualizado en las fases espiratorias $(32,33)$

Finalmente, se pueden ver cambios fibróticos que desarrollan hasta un $20 \%$ de los pacientes, dentro de los cuales se encuentran la presencia de bandas septales gruesas, distorsión de la arquitectura, pérdida de volumen, quistes en el panal de abejas y bronquiectasias de tracción, hallazgos indicativos de fibrosis irreversible (34). Son más frecuentes en los campos pulmonares medios y superiores, con una distribución parcheada. En casos avanzados se pueden asociar a una hipertensión pulmonar y/o insuficiencia cardiaca derecha. Otras manifestaciones radiológicas menos frecuentes no se abordarán en la presente revisión.

\section{OTRAS MANIFESTACIONES CLÍNICAS}

El compromiso ocular por sarcoidosis está descrito como el tercero más frecuente, en algunas series entre el 10-60\% de los pacientes (35). Produce una inflamación no solo ocular también de los anexos, presentándose más comúnmente en mujeres afrodescendientes.

La uveítis es el compromiso más nombrado, se da de manera heterogénea y es más prevalente en la población japonesa, donde se reporta hasta en $15 \%$ de los pacientes. Su presentación tiene dos picos, el primero de ellos en la tercera década (asociado generalmente con las formas agudas) y el segundo en la sexta o séptima (asociado a las formas crónicas). Sus síntomas pueden incluir lagrimeo, fotofobia, dolor e inyección conjuntival. Sin embargo, alrededor de un tercio de los pacientes con uveítis causada por sarcoidosis no tiene síntomas oculares $(35,36)$. Tiende a ser bilateral $y$ tiene preferencia por el segmento anterior en un 70 al $85 \%$ de las veces con panuveítis del 9-30\% de los casos, siendo este último un compromiso grave que lleva a la ceguera en un $10 \%$ de los pacientes, generalmente en el transcurso del primer año después de 
su debut $(35,37)$. El segundo compromiso más usual es el de las conjuntivas, observándose generalmente como nódulos (37). Otras formas menos usuales son la vasculitis retiniana, el síndrome de Heerfordt (descrito previamente) y la proptosis ocular $(38,39)$, haciendo énfasis en que cualquier segmento ocular puede verse afectado por esta enfermedad.

La sarcoidosis cutánea se presenta como primer síntoma entre el 20-35\% de los pacientes, puede ser una forma limitada a este óroano en el 70 \% de los casos. Existen presentaciones cutáneas específicas que requieren la presencia de granulomas no caseificantes en la biopsia, y otras denominadas no específicas que no cumplen esta condición. La forma más frecuente reportada en la literatura es el eritema nodoso, el cual hace parte de las lesiones inespecíficas, además de calcificaciones, acropaquia, pioderma gangrenoso y síndrome de Sweet (40).

Las lesiones cutáneas específicas están presentes entre el 9-15\% de los pacientes con sarcoidosis, representadas por pápulas y placas, el lupus pernio (placas violáceas), el cual no debe confundirse con lupus cutáneo. Esta lesión se produce generalmente en la nariz y las mejillas, confiere un mal pronóstico porque predice un mayor riesgo de progresión a las formas crónicas de la enfermedad y a manifestaciones pulmonares.

Otras formas incluyen la sarcoidosis en cicatrices, tatuajes y los nódulos subcutáneos o sarcoidosis de Darier Roussy (inflamación subcutánea dada por nódulos) entre otras (40).

La manifestación articular por sarcoidosis puede simular artropatías agudas o crónicas, se presenta en el $25 \%$ de los pacientes durante el curso de la enfermedad. En la serie de Muñoz et al. se encontró una elevada presencia de artritis en tobillos $62 \%$ y articular en el $59 \%$ de los casos (9), diferente a lo reportado en otras series colombianas donde la mitad fueron hombres y el compromiso articular fue mucho menor, cercano al 8 \% (40); esta diferencia se explica principalmente por sesgos de referencia de los mencionados estudios (41). La artritis bilateral del tobillo es característica de esta enfermedad, la cual tiene una sensibilidad del $95 \%$ y una especificidad del $90 \%$ en casos con sospecha de sarcoidosis $(42,43)$.

La prevalencia de sarcoidosis del sistema nervioso se reporta entre el 3,5-7 \%, siendo la neuropatía craneal la más común (47-64\%), seguido por el sistema nervioso central entre el 14-64\% de los pacientes y las manifestaciones periféricas 3-14\% (43). En aquellos pacientes con enfermedad sistémica y síntomas neurológico, el $60 \%$ coincide con el inicio, el $34 \%$ lo precede y en el $6 \%$ se da posterior al inicio de los síntomas (44).

La sarcoidosis renal es poco frecuente y es usualmente asintomática (90\% de los casos) (45), siendo el $10 \%$ restante la formas sintomáticas y la más asociada la nefritis intersticial (45). La sarcoidosis cardiaca clínicamente evidente se ha descrito entre el 2-7 \% de los pacientes, reportándose el compromiso oculto en más del $20 \%$ de los casos; es importante resaltar que la muerte súbita puede ser la manifestación inicial (dado por trastornos del ritmo o de la conducción), pudiendo presentarse en cualquier momento de la enfermedad.

Es más característico en población japonesa con incidencias del 50 al 78 \% en estudios de necropsias, en estos la principal causa de muerte es la cardiaca (77-85\%), a diferencia de otras razas (13-50 \%) (7). Las dificultades con este forma de manifestación están dadas por el diagnóstico definitivo, pues la biopsia endomiocárdica tiene un bajo rendimiento y el diagnóstico se basa en estudios imagenológicos no invasivos; estando autorizado el tratamiento en ausencia del diagnóstico histológico (7).

La sarcoidosis hepática está subestimada, se ha descrito un 11,5 \% según el estudio ACCESS (10), pues gran parte de los pacientes son asintomáticos o sus síntomas son poco específicos, más aún si se compara el compromiso histológico y el de las pruebas hepáticas alteradas en ausencia de síntomas. La biopsia es positiva en el $75 \%$ de los pacientes, los síntomas son usualmente dolor abdominal, prurito, fiebre, ictericia y pérdida de peso. La hepatomegalia se presenta entre el 5-15\% de los pacientes y la anormalidad bioquímica más descrita es la elevación de la fosfatasa alcalina, presente en el 90 \% de los sintomáticos (46).

Los hallazgos hematológicos en pacientes con sarcoidosis son raros, se han descrito linfadenopatías periféricas, anemia, trombocitopenia, anemia de enfermedad crónica, linfopenia y leucopenia, dichos hallazgos parecen ser por granulomas en la médula ósea o por el secuestro esplénico (47).

\section{DIAGNÓSTICO}

Como enfermedad su diagnóstico es desafiante, pues es eminentemente de descarte (48). Para esto se requiere 
de tres condiciones: la presencia de granulomas sin necrosis de caseificación (idealmente, en al menos dos órganos) (18), descartando por enfermedades con fenotipo similar y un cuadro clínico compatible (48). Al momento de iniciar con el proceso de diagnóstico, el primer paso es definir si se tiene un compromiso multisistémico, luego se debe descartar la presencia de alguna de las condiciones en las que se puede realizar el diagnóstico de sarcoidosis sin necesidad de biopsia (como en el síndrome de Löfogren y de Heerfordt) o en presencia de: adenopatías hiliares bilaterales asintomáticas en presencia del signo del panda y el signos de lambda en el rastreo con galio-67 (18).

Posteriormente se buscará la confirmación histológica dando prioridad a las lesiones sospechosas de fácil acceso y en caso de no ser posible, se definirá la biopsia de adenopatías mediastinal o hiliares. Finalmente, si no existen lesiones susceptibles, puede ser de utilidad realizar una biopsia a ciegas en ciertos tejidos como conjuntiva con $55 \%$ de positividad (49), glándula salival $(20-58 \%)(50,51)$, ganglio linfático escaleno (74-80 \%) (52) e hígado (50-60 \%) (53).

Históricamente, se ha descrito el aumento de los niveles de la enzima convertidora de angiotensina (ECA) en el diagnóstico de la enfermedad (54). El origen de esta asociación no es del todo claro y pareciera estar en relación con un mayor riesgo de que algunos genotipos de la ECA confieren mayor riesgo de presentar la enfermedad. Su sensibilidad y especificidad se han calculado en el $41,4 \%$ y $89,9 \%$, respectivamente, con una tasa de falsos positivos alrededor de un 10 $\%$, por lo que como prueba única en el diagnóstico de esta enfermedad su uso no es aconsejable $(15,54)$.

Si bien estas limitaciones son importantes en pacientes en quienes el diagnóstico diferencial está enfocado en otras enfermedades granulomatosas, la elevación dos veces por encima del límite superior es altamente específica de sarcoidosis y allí podría residir su mayor utilidad clínica (55). Otros aspectos que pueden afectar su rendimiento son la hiperactividad familiar de la ECA, que se asocia con niveles elevados por encima de tres veces el valor usual de referencia en las personas sanas (56) y el tratamiento con esteroides que pueden disminuir los valores de esta. Por otra parte, la utilidad en medir los niveles de ECA de forma seriada para determinar la actividad de la enfermedad no ha sido demostrada $(55,57)$. Otros exámenes que pueden dar información importante incluyen la tuberculina, que en pacientes con sarcoidosis activa es generalmente negativa por enerớa periférica, siendo necesario aclarar que al tener tuberculina positiva se debe hacer búsqueda de tuberculosis como causa de los síntomas (58).

Algunos exámenes que han caído en desuso incluyen el test de Kveim Siltzbachm, en el cual se utilizaba tejido, preferentemente del bazo, de un paciente con sarcoidosis y se aplicaba de forma subcutánea. Posteriormente se tomaba una biopsia del sitio de la lesión, cuatro a seis semanas más tarde, y se observaba la formación de granulomas. Si bien esta prueba presentaba una sensibilidad alta (87\%) para el diagnóstico de sarcoidosis, las dificultades para obtener la materia prima por la pobre disponibilidad de esta la han relegado a ser una curiosidad histórica (59).

Otro estudio que se ha realizado de forma rutinaria es la gammagrafía marcada con galio-67, este examen da una captación característica en glándulas parótidas y lacrimales "signo del panda" y en la región mediastinal con compromiso paratraqueal y pretraqueal "signo de lambda”. La presencia de estas alteraciones es altamente específica de sarcoidosis, no obstante, por su baja sensibilidad y pobre disponibilidad no es un examen utilizado de forma común en la práctica clínica (60).

Finalmente, existen algunas innovaciones en el campo de los modelos diagnósticos para tratar de homogeneizar este proceso, en una enfermedad como esta sin hallazoos patognomónicos. Por esto la Asociación Mundial de Sarcoidosis y otras Enfermedades Granulomatosas (World Association of Sarcoidosis and other Granulomatous Disorders, WASOG) desarrolló una lista de evaluación de afección de órganos por sarcoidosis, clasificándolos en altamente probables, probable, posible y no definible (61).

Este instrumento brinda al clínico una mejor evaluación, aunque no da un puntaje que permita tomar una decisión y que provea una sensibilidad y especificidad conocida. Con base en esa lista, de forma reciente se ha desarrollado un puntaje diagnóstico de sarcoidosis que tiene en cuenta dos brazos, el clínico y el histológico, además de proveer puntos de corte con una sensibilidad conocida. De este modo, un puntaje de 6 o más en la rama de biopsia resulta en una sensibilidad del 99,3 \% y una especificidad del $100 \%$; y un puntaje de 3 en la rama clínica tiene una sensibilidad del $94,2 \%$ y especificidad del $88,8 \%$, que cambia a $76,9 \%$ y $98,6 \%$, respectivamente, si el punto de corte se modifica a 4 . 
Si bien estos hallazgos aún no han sido validados por otros grupos, podrían permitir una aproximación mucho más amigable para el clínico (62).

\section{DIAGNÓSTICO DIFERENCIAL}

Entre sus diagnósticos diferenciales es necesario siempre tener en cuenta causas infecciosas, especialmente tuberculosis, pues esta enfermedad es común en nuestro medio (prevalencia de 24,1/100.000 para 2013) y produce lesiones granulomatosas. Entre otras infecciones a tener en cuenta se incluyen algunas micóticas como histoplasma, que también pueden generar granulomas (63).

Usualmente el diagnóstico diferencial requiere un enfoque multidimensional donde el hallazgo histológico debe ser evaluado mediante tinción y cultivo para descartar causas infecciosas (64). Las vasculitis asociadas a anticuerpos contra el citoplasma de los neutrófilos (ANCA), sobre todo la granulomatosis con poliangeitis, son un diferencial obligado, pues induce necrosis sin caseificación, compromiso pulmonar, ocular y de otros órganos, donde la presencia de vasculitis en la biopsia será una de las formas de diferenciarlo. Existen otros detalles en la evolución como el compromiso renal, típico de este tipo de vasculitis, y la positividad de los ANCA, especialmente por ELISA, que son particularmente útiles para realizar esta diferenciación (14).

La inmunodeficiencia común variable es otro diagnóstico a tener en cuenta, pues la enfermedad pulmonar granulomatosa con infiltración linfocítica asociada a la inmunodeficiencia común variable puede ser incorrectamente diagnosticada como sarcoidosis. Se trata de una enfermedad sistémica, con linfadenopatías, esplenomegalia, granulomatosis extra e intrapulmonar, con necrosis sin caseificación. En este caso, la hipogammaglobulinemia y la región gamma plana en la electroforesis de proteínas orientan el diagnóstico. por último, los hallazgos pulmonares difieren de manera importante a pesar de las similitudes en la biopsia, siendo más frecuente la presencia de vía superior y las adenopatías en sarcoidosis (65).

El síndrome del Sjögren hace parte del diagnóstico diferencial de los pacientes con sarcoidosis, pues la parotidomegalia, el compromiso de glándulas salivales y lagrimales hace parte de las manifestaciones descritas de la enfermedad. En este síndrome hay autoinmunidad de las glándulas exocrinas, produciendo síntomas como boca seca y ojos secos. Se han descrito la coexistencia de ambas enfermedades, como en la serie de casos de Ramos-Casals (66), en esta se detalla en que ambas enfermedades comparten, clínica, inmunogenética y patogenia, lo que ayuda a diferenciar es la presencia de anticuerpos el Sjögren, usualmente negativos en sarcoidosis, además de la biopsia de glándula salival donde el predominio de CD8+ en sarcoidosis y, CD4+ en Sjögren hará la diferencia, incluso ante las dudas en los hallazgos histológicos típicos que se pueden superponer (66).

En tiempos recientes, se ha descrito la enfermedad por IogG4 como multisistémica, caracterizada por el compromiso fibroinflamatorio de múltiples órganos: retroperitoneo, glándulas salivares, páncreas, ganglios, cerebro, piel, grandes vasos, entre otros. Manifestado como pancreatitis, colangitis esclerosante, nefritis intersticial, prostatitis, neumonía y aumento de las glándulas salivares (67).

Las características mencionadas hacen difícil diferenciar estas dos enfermedades, a veces se trata de manera errónea la IgG4, bajo el supuesto diagnóstico de sarcoidosis (68) e incluso hay reportes en la literatura de pancreatitis por IgG4, con compromiso granulomatoso en ganglios mediastinales y pulmón, donde se plantea la coexistencia de ambas (69). Ante esta difícil situación, se ha propuesto la gammagrafía con galio-67 como herramienta útil en el diagnóstico diferencial, mediante la captación en páncreas, glándulas submandibular (especialmente si es asimétrica) en enfermedad por IgG4, mientras en la sarcoidosis este predomina en glándulas lacrimales, adenopatías supraclaviculares, mediastinales y el tejido muscular (70).

Si bien no es el propósito de esta revisión, el tratamiento es variable desde la observación clínica en las formas asintomáticas hasta el tratamiento inmunosupresor completo con agentes alquilantes en las formas más graves. Adicionalmente, en casos refractarios se han utilizado anti-TNF como alternativa, lo cual tiene lógica por el efecto lítico de estos sobre los granulomas (71).

En conclusión, la sarcoidosis es una enfermedad granulomatosa multisistémica de difícil identintificacion. Su diagnóstico diferencial es amplio y requiere de un proceso metódico de descarte, pues no existe una prueba diagnóstica definitiva. Finalmente, el 
tratamiento depende del grado de compromiso orgánico y la cronicidad de esta.

\section{CONFLICTOS DE INTERESES}

Ninguno por declarar.

\section{REFERENCIAS BIBLIOGRÁFICAS}

1. Spagnolo P, Luppi F, Roversi P, Cerri S, Fabbri LM, Richeldi L. Sarcoidosis: challenging diagnostic aspects of an old disease. Am J Med. 2012 Feb;125(2):118-25. DOI 10.1016/j.amjmed.2011.06.003.

2. Johns CJ, Michele TM. The clinical management of sarcoidosis. A 50-year experience at the Johns Hopkins Hospital. Medicine (Baltimore). 1999 Mar;78(2):65-111.

3. Hem E. [Multiple benign sarcoid of the skin--100 years since Caesar Boeck's pioneering article]. Tidsskr Nor Laegeforen. 1999 Dec;119(30):4567-9.

4. Iannuzzi MC, Fontana JR. Sarcoidosis: clinical presentation, immunopathogenesis, and therapeutics. JAMA. 2011 Jan 26;305(4):391-9. DOI 10.1001/jama.2011.10.

5. Westney GE, Judson MA. Racial and ethnic disparities in sarcoidosis: from genetics to socioeconomics. Clin Chest Med. 2006 Sep;27(3):453-62, vi.

6. Iannuzzi MC, Rybicki BA, Teirstein AS. Sarcoidosis. N Engl J Med. 2007 Nor;357(21):2153-65.

7. Lynch JP 3rd, Hwang J, Bradfield J, Fishbein M, Shivkumar K, Tung R. Cardiac involvement in sarcoidosis: evolving concepts in diagnosis and treatment. Semin Respir Crit Care Med. 2014 Jun;35(3):372-90. DOI 10.1055/s-0034-1376889.

8. Santamaria-Alza Y, Fajardo Rivero JE. Sarcoidosis: una serie de casos del nororiente colombiano. Neumol Cir Tórax. 2017 Ene-Mar;76(1):14-6.

9. Muñoz C, Restrepo-Escobar M, Martínez-Muñoz M, Echeverri A, Márquez J, Pinto LF. Differences Between Patients With Sarcoidosis With and Without Joint Involvement Treated for Fifteen Years in a Third Level Hospital. Reumatol Clin. 2018 Feb. Pii: S1699258X(18)30021-4. DOI 10.1016/j.reuma.2018.01.001.

10. Judson MA, Baughman RP, Thompson BW, Teirstein AS, Terrin ML, Rossman MD, et al. Two year prognosis of sarcoidosis: the ACCESS experience. Sarcoidosis Vasc Diffuse Lung Dis. 2003 Oct;20(3):204-11.
11. Co DO, Hogan LH, II-Kim S, Sandor M. T cell contributions to the different phases of granuloma formation. Immunol Lett. 2004 Mar;92(1-2):135-42.

12. Song Z, Marzilli L, Greenlee BM, Chen ES, Silver RF, Askin $\mathrm{FB}$, et al. Mycobacterial catalase-peroxidase is a tissue antigen and target of the adaptive immune response in systemic sarcoidosis. J Exp Med. 2005 Mar;201(5):755-67. Erratum in: J Exp Med. 2005 Sep 5;202(5):721.

13. Baughman RP, Teirstein AS, Judson MA, Rossman MD, Yeager H Jr, Bresnitz EA, et al. Clinical characteristics of patients in a case control study of sarcoidosis. Am J Respir Crit Care Med. 2001 Nov;164(10 Pt 1):1885-9.

14. Rybicki BA, Major M, Popovich J Jr, Maliarik MJ, Iannuzzi MC. Racial differences in sarcoidosis incidence: a 5-year study in a health maintenance organization. Am J Epidemiol. 1997 Feb;145(3):234-41.

15. Carmona EM, Kalra S, Ryu JH. Pulmonary Sarcoidosis: Diaognosis and Treatment. Mayo Clin Proc. 2016 JuI;91(7):946-54. DOI 10.1016/j.mayocp.2016.03.004.

16. Hamzeh N. Sarcoidosis. Med Clin North Am. 2011 Nov;95(6):1223-34. DOI 10.1016/j.mcna.2011.08.004.

17. Chen ES, Moller DR. Etiology of sarcoidosis. Clin Chest Med. 2008 Sep;29(3):365-77, vii. DOI 10.1016/j. ccm.2008.03.011.

18. Haimovic A, Sanchez M, Judson MA, Prystowsky S. Sarcoidosis: a comprehensive review and update for the dermatologist: part I. Cutaneous disease. J Am Acad Dermatol. 2012 May;66(5):699.e1-18; quiz 717-8. DOI 10.1016/j.jaad.2011.11.965.

19. Govender P, Berman JS. The Diagnosis of Sarcoidosis. Clin Chest Med. 2015 Dec;36(4):585-602. DOI 10.1016/j.ccm.2015.08.003.

20. Chappity P, Kumar R, Sahoo AK. Heerfordt's Syndrome Presenting with Recurrent Facial Nerve Palsy: Case report and 10-year literature review. Sultan Qaboos Univ Med J. 2015 Feb;15(1):e124-8.

21. Baughman RP. Pulmonary sarcoidosis. Clin Chest Med. 2004 Sep;25(3):521-30, vi.

22. Statement on sarcoidosis. Joint Statement of the American Thoracic Society (ATS), the European Respiratory Society (ERS) and the World Association of Sarcoidosis and Other Granulomatous Disorders (WASOG) adopted by the ATS Board of Directors and by the ERS Executive Committee, February 1999. Am J Respir Crit Care Med. 1999 Aug; 160(2):736-55. 
23. Judson MA. The Clinical Features of Sarcoidosis: A Comprehensive Review. Clin Rev Allergy Immunol. 2015 Aug;49(1):63-78. DOI 10.1007/s12016-0148450-y.

24. Hillerdal G, Nöu E, Osterman K, Schmekel B. Sarcoidosis: epidemiology and prognosis. A 15year European study. Am Rev Respir Dis. 1984 Jul;130(1):29-32.

25. Siltzbach LE, James DG, Neville E, Turiaf J, Battesti JP, Sharma OP, et al. Course and prognosis of sarcoidosis around the world. Am J Med. 1974 Dec;57(6):847-52.

26. Lynch JP 3rd, Kazerooni EA, Gay SE. Pulmonary sarcoidosis. Clin Chest Med. 1997 Dec;18(4):755-85.

27. Baughman RP, Winget DB, Bowen EH, Lower EE. Predicting respiratory failure in sarcoidosis patients. Sarcoidosis Vasc Diffuse Lung Dis. 1997 Sep;14(2):154-8.

28. Nishimura $K$, Itoh $H$, Kitaichi $M$, Nagai $S$, Izumi T. CT and pathological correlation of pulmonary sarcoidosis. Semin Ultrasound CT MR. 1995 Oct; 16(5):361-70.

29. Brauner MW, Grenier P, Mompoint D, Lenoir S, de Crémoux H. Pulmonary sarcoidosis: evaluation with highresolution CT. Radiology. 1989 Aug; 172(2):467-71.

30. Müller NL, Miller RR. Ground-glass attenuation, nodules, alveolitis, and sarcoid granulomas. Radiology. 1993 Oct;189(1):31-2.

31. Hamper UM, Fishman EK, Khouri NF, Johns CJ, Wang KP, Siegelman SS. Typical and atypical CT manifestations of pulmonary sarcoidosis. J Comput Assist Tomogr. 1986 Nov-Dec;10(6):928-36.

32. Reich JM. Mortality of intrathoracic sarcoidosis in referral $\nabla$ s population-based settings: influence of stage, ethnicity, and corticosteroid therapy. Chest. 2002 Jan; 121(1):32-9.

33. Davies CW, Tasker AD, Padley SP, Davies RJ, Gleeson FV. Air trapping in sarcoidosis on computed tomography: correlation with lung function. Clin Radiol. 2000 Mar;55(3):217-21.

34. Bartz RR, Stern EJ. Airways obstruction in patients with sarcoidosis: expiratory CT scan findings. J Thorac Imaging. 2000 Oct;15(4):285-9.

35. Abehsera M, Valeyre D, Grenier P, Jaillet H, Battesti JP, Brauner MW. Sarcoidosis with pulmonary fibrosis: CT patterns and correlation with pulmonary function. AJR Am J Roentgenol. 2000 Jun;174(6):1751-7.
36. Jamilloux X, Kodjikian L, Broussolle C, Sève P. Sarcoidosis and uveitis. Autoimmun Rev. 2014 Aug; 13(8):8409. DOI 10.1016/j.autrev.2014.04.001.

37. Martínez-Berriotxoa A, Fonollosa A, Artaraz J. [Uveitis: diagnostic approach]. Rev Clin Esp. 2012 Oct;212(9):442-52. DOI 10.1016/j.rce.2011.12.004.

38. Rao DA, Dellaripa PF. Extrapulmonary manifestations of sarcoidosis. Rheum Dis Clin North Am. 2013 May;39(2):277-97. DOI 10.1016/j.rdc.2013.02.007.

39. Kansal $\nabla$, Dollin M. Ocular involvement in sarcoidosis. CMAJ. 2017 Apr;189(16):E609. DOI 10.1503/ cmaj. 160569.

40. Zapata-González F, Vásquez-Ochoa LA, ArroyaveSierra JE, Arredondo-Ossa MI, Molina-Vélez V, RíoCobaleda D. Sarcoidosis cutánea. CES Med. 2014 JulDic;28(2):293-306.

41. Valovis R. Sarcoidosis estudio clínico de 51 casos y revisión de la literatura. Acta Médica Colomb. 1977;2(2):101-10.

42. Visser H, Vos K, Zanelli E, Verduyn W, Schreuder GM, Speyer I, et al. Sarcoid arthritis: clinical characteristics, diagnostic aspects, and risk factors. Ann Rheum Dis. 2002 Jun;61(6):499-504.

43. Banse $C$, Goëb $\nabla$. Do not forget the joint involvement of sarcoidosis. Immunotherapy. 2015;7(6):599-600. DOI 10.2217/imt.15.24.

44. Kidd DP. Sarcoidosis of the central nervous system: clinical features, imaging, and CSF results. J Neurol. 2018 Aug;265(8):1906-15. DOI 10.1007/s00415-018-8928-2.

45. Rocha ÓG, García PK, Echeverri JE, D’Achiardi RE, Rodríguez MP, Córdoba JP, et al. Sarcoidosis y compromiso renal: reporte de un caso y revisión de la literatura científica. Univ Méd. 2012 Ene-Mar;53(1):94-106.

46. Holmes J, Lazarus A. Sarcoidosis: extrathoracic manifestations. Dis Mon. 2009 Nor;55(11):675-92. DOI 10.1016/j.disamonth.2009.05.002.

47. Okumus G, Musellim B, Cetinkaya E, Turker H, Uzaslan E, Yenturk E, et al. Extrapulmonary involvement in patients with sarcoidosis in Turkey. Respirology. 2011 Apr;16(3):446-50. DOI 10.1111/j.1440-1843.2010.01878.x.

48. Valeyre D, Bernaudin JF, Uzunhan Y, Kambouchner M, Brillet PY, Soussan M, et al. Clinical presentation of sarcoidosis and diagnostic work-up. Semin Respir Crit Care Med. 2014 Jun;35(3):336-51. DOI 10.1055/s-00341381229. 
49. Bonfioli AA, Orefice F. Sarcoidosis. Semin Ophthalmol. 2005 Jul-Sep;20(3):177-82.

50. Harvey J, Catoggio L, Gallagher PJ, Maddison PJ. Salivary gland biopsy in sarcoidosis. Sarcoidosis. 1989 Mar;6(1):47-50.

51. Blaise P, Fardeau C, Chapelon C, Bodaghi B, Le Hoang P. Minor salivary gland biopsy in diagnosing ocular sarcoidosis. Br J OphthaImol. 2011 Dec;95(12):1731-4. DOI 10.1136/bjophthalmol-2011-300129.

52. Truedson H, Stjernberg N, Thunell M. Scalene lymph node biopsy. A diagnostic method in sarcoidosis. Acta Chir Scand. 1985;151(2):121-3.

53. Karagiannidis A, Karavalaki M, Koulaouzidis A. Hepatic sarcoidosis. Ann Hepatol. 2006 Oct-Dec;5(4):251-6.

54. Ungprasert P, Carmona EM, Crowson CS, Matteson EL. Diagnostic Utility of Angiotensin-Converting Enzyme in Sarcoidosis: A Population-Based Study. Lung. 2016 Feb;194(1):91-5. DOI 10.1007/s00408-015-9826-3.

55. Lieberman J. Elevation of serum angiotensin-converting-enzyme (ACE) level in sarcoidosis. Am J Med. 1975 Sep;59(3):365-72.

56. Stouten K, van de Werken M, Tchetverikov I, Saboerali M, Vermeer HJ, Castel R, et al. Extreme elevation of serum angiotensin-converting enzyme (ACE) activity: always consider familial ACE hyperactivity. Ann Clin Biochem. 2014 Mar;51(Pt 2):289-93. DOI 10.1177/0004563213489812.

57. Studdy PR, Bird R. Serum angiotensin converting enzyme in sarcoidosis-its value in present clinical practice. Ann Clin Biochem. 1989 Jan;26 ( Pt 1):13-8.

58. Gupta D, Chetty M, Kumar N, Aggarwal AN, Jindal SK. Anergy to tuberculin in sarcoidosis is not influenced by high prevalence of tuberculin sensitivity in the population. Sarcoidosis Vasc Diffuse Lung Dis. 2003 Mar;20(1):40-5.

59. Siltzbach LE. The Kveim test in sarcoidosis. A study of 750 patients. JAMA. 1961 Nov;178:476-82.

60. Sulavik SB, Spencer RP, Palestro CJ, Swyer AJ, Teirstein AS, Goldsmith SJ. Specificity and sensitivity of distinctive chest radiographic and/or 67Ga images in the noninvasive diagnosis of sarcoidosis. Chest. 1993 Feb;103(2):403-9.
61. Judson MA, Costabel U, Drent M, Wells A, Maier L, Koth L, et al. The WASOG Sarcoidosis Organ Assessment Instrument: An update of a previous clinical tool. Sarcoidosis Vasc Diffuse Lung Dis. 2014 Apr;31(1):19-27.

62. Bickett AN, Lower EE, Baughman RP. Sarcoidosis Diagnostic Score: A Systematic Evaluation to Enhance the Diagnosis of Sarcoidosis. Chest. 2018 Nov;154(5):1052-60. DOI 10.1016/j.chest.2018.05.003.

63. Newman LS, Rose CS, Maier LA. Sarcoidosis. N Engl J Med. 1997 Apr;336(17):1224-34. Erratum in: N Engl J Med 1997 JuI;337(2):139.

64. Judson MA. The diagnosis of sarcoidosis. Clin Chest Med. 2008 Sep;29(3):415-27. DOI 10.1016/j. ccm.2008.03.009.

65. Verbsky JW, Routes JM. Sarcoidosis and common variable immunodeficiency: similarities and differences. Semin Respir Crit Care Med. 2014 Jun;35(3):3305. DOI 10.1055/s-0034-1376862.

66. Ramos-Casals M, Brito-Zerón P, García-Carrasco M, Font J. Sarcoidosis or Sjögren syndrome? Clues to defining mimicry or coexistence in 59 cases. Medicine (Baltimore). 2004 Mar;83(2):85-95.

67. Yu JQ, Doss M, Codreanu I, Zhuang H. PET/CT in Patients with Sarcoidosis or IgG4 Disease. PET Clin. 2012 Apr;7(2):191-210. DOI 10.1016/j.cpet.2012.01.005.

68. Yu J, Hall T, Kale S, Phillips A, Madge S. IgG4 disease: a revised diagnosis of sarcoidosis after 36 years of treatment. Br J Hosp Med (Lond). 2013 Aug; 74(8):470-1.

69. Michel L, Clairand R, Néel A, Masseau A, Frampas E, Hamidou M. Association of IgG4-related disease and sarcoidosis. Thorax. 2011 Oct;66(10):920-1. DOI 10.1136/thx.2011.160341.

70. Ishii S, Miyajima M, Sakuma K, Kikuchi K, Shishido F. Comparison between sarcoidosis and IgG4-related disease by whole-body $67 \mathrm{Ga}$ scintigraphy. Nucl Med Commun. 2013 Jan;34(1):13-8. DOI 10.1097/ MNM.obo13e32835a2eea.

71. Baughman RP, Grutters JC. New treatment strategies for pulmonary sarcoidosis: antimetabolites, biological drugs, and other treatment approaches. Lancet Respir Med. 2015 Oct;3(10):813-22. DOI 10.1016/ S2213-2600(15)00199-X. 